\title{
GCU
}

Glasgow Caledonian

University

University for the Common Good

\section{A colour preference technique to evaluate acrylamide-induced toxicity in zebrafish}

Jia, Laibing ; Raghupathy, Rakesh Kotapati; Albalawi, Aishah; Zhao, Zhenkai ; Reilly, James; Xiao, Qing ; Shu, Xinhua

Published in:

Comparative Biochemistry and Physiology Part C: Toxicology \& Pharmacology

DOI:

10.1016/j.cbpc.2017.01.004

Publication date:

2017

Document Version

Author accepted manuscript

Link to publication in ResearchOnline

Citation for published version (Harvard):

Jia, L, Raghupathy, RK, Albalawi, A, Zhao, Z, Reilly, J, Xiao, Q \& Shu, X 2017, 'A colour preference technique to evaluate acrylamide-induced toxicity in zebrafish', Comparative Biochemistry and Physiology Part C: Toxicology \& Pharmacology, vol. 199, no. 2017, pp. 11-19. https://doi.org/10.1016/j.cbpc.2017.01.004

\section{General rights}

Copyright and moral rights for the publications made accessible in the public portal are retained by the authors and/or other copyright owners and it is a condition of accessing publications that users recognise and abide by the legal requirements associated with these rights.

Take down policy

If you believe that this document breaches copyright please view our takedown policy at https://edshare.gcu.ac.uk/id/eprint/5179 for details of how to contact us. 
1 A colour preference technique to evaluate acrylamide-induced toxicity in zebrafish

2 Laibing Jia ${ }^{1,3^{*}}$, Rakesh Kotapati Raghupathy ${ }^{2 *}$, Aishah Albalawi ${ }^{2}$, Zhenkai Zhao ${ }^{1}$, James

3 Reilly $^{2}$, Qing Xiao ${ }^{1 \#}, X^{\prime n h u a ~ S h u}{ }^{2 \#}$

4 1. Department of Naval Architecture, Ocean, and Marine Engineering, University of

5 Strathclyde, Glasgow G4 0LZ, United Kingdom

6 2. Department of Life Sciences, Glasgow Caledonian University, Glasgow G4 0BA, United

7 Kingdom

8 3. School of Marine Science and Technology, Northwestern Polytechnical University, Xi'an,

$9 \quad$ Shaanxi, 710072, China

*LJ and RKR made equal contribution.

\# Corresponding authors: Xinhua Shu, Department of Life Sciences, Glasgow Caledonian University, Glasgow G4 0BA, UK, E-mail: Xinhua.Shu@gcu.ac.uk; Qing Xiao, Department of Naval Architecture, Ocean, and Marine Engineering, University of Strathclyde, Glasgow G4 0LZ, UK, Email: qing.xiao@strath.ac.uk. 


\section{ABSTRACT}

27 The zebrafish has become a commonly used vertebrate model for toxicity assessment, of particular relevance to the study of toxic effects on the visual system because of the structural similarities shared by zebrafish and human retinae. In this article we present a colour preference-based technique that, by assessing the functionality of photoreceptors, can be used to evaluate the effects of toxicity on behaviour. A digital camera was used to record the locomotor behaviour of individual zebrafish swimming in a water tank consisting of two compartments separated by an opaque perforated wall through which the fish could pass. The colour of the lighting in each compartment could be altered independently (producing distinct but connected environments of white, red or blue) to allow association of the zebrafish's swimming behaviour with its colour preference. The functionality of the photoreceptors was evaluated based on the ability of the zebrafish to sense the different colours and to swim between the compartments. The zebrafish tracking was carried out using our algorithm developed with MATLAB. We found that zebrafish preferred blue illumination to white, and white illumination to red. Acute treatment with acrylamide ( $2 \mathrm{mM}$ for 36 hours) resulted in a marked reduction in locomotion and a concomitant loss of colour-preferential swimming behaviour. Histopathological examination of acrylamide-treated zebrafish eyes showed that acrylamide exposure had caused retinal damage. The colour preference tracking technique 
has applications in the assessment of neurodegenerative disorders, as a method for preclinical appraisal of drug efficacy and for behavioural evaluation of toxicity.

KEY WORDS Zebrafish; vision; colour preference; photoreceptors; acrylamide; toxicity

\section{Introduction}

The zebrafish has become a popular animal model in the field of neuroscience and developmental biology (Kabashi et al., 2011; Newman et al., 2011). Recent developments in transgenic technology and reverse genetic approaches have resulted in the zebrafish becoming one of the animal models most commonly used to study human health and dysfunction, including neurodegenerative disorders such as Alzheimer's disease, Parkinson's disease and, in particular, retinal disorders (Fadool \& Dowling, 2008). Various transgenic and mutant zebrafish strains mimicking human disease conditions have helped to elucidate the function and mechanisms of many proteins, while a variety of chemicals have been used to help understand the pathology of particular diseases and to develop strategies for therapy.

Visual behavioural techniques such as optokinetic response (OKR) and optomotor response (OMR) have been useful in studying defects in motion perception, visual processing, light sensing and, to some extent, colour vision; these techniques are currently used in screening genetically modified animals and assessing their visual performance after genetic and pharmacological modifications. These methods are used mainly with larval fish in the assessment of visual performance based on motion cues, but since most human ocular disorders have a late onset there is a need for visual behavioural tests that can be used to study adult fish (Fleisch \& Neuhauss, 2006). Studies carried out in recent years have 
introduced assays of zebrafish learning and behaviour that have been used in testing the neurotoxicity of drugs (Avdesh et al., 2012; Bault et al. 2015; Emran et al., 2008; Li et al., 2014; Parng et al., 2007; Schloss, 2015). Natural colour preference is a major factor by which learning, memory, decision-making and functionality of the zebrafish visual system can be assayed.

The zebrafish retina is tetrachromatic, containing (in addition to the rod photoreceptor) four different types of cone cells capable of processing four regions of the colour spectrum, namely red, green, blue and ultraviolet (Raghupathy et al., 2013). Due to the complex functionality of its photoreceptors, determining the specific functionality of individual zebrafish cone photoreceptor types using conventional molecular techniques is a difficult and laborious process. Behavioural techniques therefore offer an alternative means of assessing retinal functionality. Zebrafish behaviour is influenced by different levels of ambient illumination and colour. Avdesh et al. (2012) used two procedures (place preference and Tmaze) to test the preference of zebrafish for four different colours: red, yellow, green and blue. In the place preference procedure the fish demonstrated an equal preference for red, yellow and green, and less preference for blue; in the T-maze procedure blue was again the least preferred colour, although with this technique the fish showed an equal preference for red and green over yellow.

In the present study, we have investigated the colour preference of zebrafish for white (mix of wavelengths), red and blue colours. We found AB strain adult zebrafish preferred blue colour to white and red and preferred white to red. We also assessed the effect of treatment with acrylamide on zebrafish colour preference behaviour. Individual fish were tracked in a tank using an algorithm developed with MATLAB, modified from the algorithm initially proposed by Pérez-Escudero et al. (2014). The functionality of different types of photoreceptors was evaluated based on the zebrafish ability to sense different colours and to 
94 demonstrate colour preferences by swimming between the compartments of the tank from one area of coloured illumination to another. This methodology based on monitoring of locomotor behaviour enables analysis of the functionality of the photoreceptors of living zebrafish and, no less importantly, provides a platform for toxicity assessment and preclinical drug development.

\section{Materials and methods}

\subsection{Ethics}

Animal work was carried out in compliance with the Animal Ethics and Welfare Committee, Department of Life Sciences, Glasgow Caledonian University, and UK Home Office under Project Licence PPL 60/4169.

\subsection{Zebrafish housing}

$\mathrm{AB}$ strain adult zebrafish were housed in 2.5 litre tanks with a maximum of 12 fish (a mixture of male and female) in each tank. Fish were maintained in $27.5-28.0^{\circ} \mathrm{C}$ water on a $14: 10$ hours light-dark cycle. The $\mathrm{pH}$ of the system water was maintained at $\sim 7.5$ and the conductivity was maintained between $300-330 \mu \mathrm{S} \mathrm{cm}^{-1}$. All the fish used in this study were between 7 and 9 months of age. Fish were fed twice daily with live shrimp and once daily with granular dry food (ZM systems, UK).

\subsection{Acrylamide treatment}

A pilot study was carried out to determine the effects of acrylamide exposure on adult zebrafish (8 month old) that were treated with acrylamide (Sigma Aldrich, UK) at $1 \mathrm{mM}$, $2 \mathrm{mM}, 3 \mathrm{mM}$ and $4 \mathrm{mM}$ concentration in system water for 72 hours. It was found that, when treated with $1 \mathrm{mM}$ acrylamide, all fish survived for 72 hours; when treated with $2 \mathrm{mM}$ 
acrylamide fish death occurred after 48 hours; and when treated with 3 or $4 \mathrm{mM}$ acrylamide, fish death occurred after 24 hours. As a result, it was decided that the behavioural study would use 36 hours exposure to $2 \mathrm{mM}$ acrylamide.

Twelve 8-month old male zebrafish (average weight $0.36 \mathrm{~g}$ and average body size $3.13 \mathrm{~cm}$ ) were transferred to a tank with system water in which acrylamide was dissolved to $2 \mathrm{mM}$ concentration; the control group consisted of eighteen 8-month old zebrafish (average weight $0.36 \mathrm{~g} \pm$ and average body size $3.13 \pm \mathrm{cm}$ ) in a tank containing only zebrafish system water. Control and acrylamide-treated zebrafish were siblings. In the treatment group, exposure to acrylamide was terminated after 36 hours by transferring the fish to acrylamide-free system water and stayed for 15 minutes; similarly zebrafish of the control group were also transferred into a tank with acrylamide-free system water and stayed for 15 minutes. The fish then underwent the behavioural study. During the treatment the fish were kept in $27.5-28.0$ ${ }^{\circ} \mathrm{C}$ water on a 14:10 hours light-dark cycle. During the behavioural tests, zebrafish were not fed. After the tests the fish were killed by immersing in 0.4\% MS222 (Sigma Aldrich, UK). The eyes were enucleated and rinsed in 1XPBS briefly. For histology the tissue was fixed in $4 \%$ PFA for 2 hours at room temperature or overnight at $4^{\circ} \mathrm{C}$.

\subsection{Experimental setup}

The experiments were carried out in a water tank made of transparent acrylic plastic sheeting with a thickness of $3 \mathrm{~mm}$. The dimensions of the tank were $230 \times 150 \times 150 \mathrm{~mm}$. It was divided into two connected compartments of equal size (115 x $150 \times 150 \mathrm{~mm})$, separated along the midline by an opaque wall perforated by five circular openings of diameter $3 \mathrm{~cm}$, one situated near each corner of the dividing wall and one situated centrally (Fig. 1). Water temperature was set at $27^{\circ} \mathrm{C}$. 

an adjustable DC power supply (CSI5003XE, Circuit Specialists, UK); the brightness was controlled by the current of the power supply. The ambient colour of each compartment of the tank was changed by placing a red or blue acrylic sheet between the LED panel light and the underside of each compartment. Only light of a specific wavelength could pass through the sheets. Absorption spectra of the red and blue acrylic sheets were measured using a Lambda $25 \mathrm{UV} /$ Vis spectrophotometer (PerkinElmer, USA). The blue filter produced a blue ambient illumination (peak wavelength $450 \mathrm{~nm}$ ) that allowed assessment of the functionality of the blue cones of the zebrafish; the red filter absorbed all wavelengths below 580nM and so produced a red ambient illumination that allowed assessment of red cone functionality. In those trials in which one of the compartments was red or blue, a neutral density sheet was placed on the underside of the white compartment in order to maintain equivalence of brightness in the two compartments. The brightness of each compartment was measured by a light meter (DT-1308, CEM, UK); in each test the brightness of the two compartments varied by less than $5 \%$. The barrier positioned across the middle of the tank allowed the zebrafish to see the colour of the other compartment through the perforations while requiring the fish to make a deliberate choice to travel from one compartment to the other. A shade was used to black out the LED panel exterior to the tank, thus ensuring that the light came only from the bottom of each compartment of the tank (Fig. 1). There was no additional illumination in the pixels) and at a frame rate of 30 frames / sec was set above the tank to capture the locomotion of the individual zebrafish.

The zebrafish was placed in the test environment for 15 mins prior to the video recording, 167 thus giving the fish time to become familiar with the conditions. Each zebrafish was placed 
into each of the two compartments on one occasion only. A single test lasted for 1000 seconds. In each condition (different colour filter pair combinations) 8 zebrafish were tested.

\subsection{Algorithm for zebrafish tracking}

The functionality of the photoreceptors was evaluated based on the zebrafish ability to sense the different colours and to demonstrate preferential behaviour by spending different amounts of time in each coloured compartment. The fish tracking was carried out using our own developed MATLAB code following the algorithm of idTracker (Pérez-Escudero, 2014).

Each captured video file of the zebrafish swimming behaviour was decoded into a sequence of images. For each frame of the video, a region of interest (ROI) mask was used to isolate, from the rest of the tank, the area currently occupied by the fish; this results in the acquisition of a subimage (the fish against its immediate background) and helps to reduce image processing time. In the subimage, there was an obvious difference in contrast between the zebrafish and its background. A histogram of light distribution within the subimage was generated. In the histogram, there were two distinct groups of light intensity: one for the zebrafish and one for the background. A threshold was determined from the mean of the two brightness distributions, this threshold value allowing differentiation of the zebrafish from the background. A binary image using the threshold was calculated from the subimage. An object detection algorithm was then applied to the binary image. By comparing the fish length, area, and other features with any other objects detected from the binary image, the zebrafish could be distinguished from other objects. Once the zebrafish was identified from the background its features (such as centroid, body orientation, centreline and tail-tip position) could be determined using our feature extraction algorithm and its positions in the video digitized. Fig. 2 shows the application of the tracking algorithm to a captured video that recorded the movement of a zebrafish between red and blue compartments. Fig. 2a shows the zebrafish in 
a consecutive sequence of frames. The zebrafish is imaged in each of the small squares and can be seen moving from the red compartment to the blue compartment. At the beginning (top left box), the fish is swimming in the "red" compartment but with time it travels through a hole in the perforated wall to the "blue" compartment. After a short period in the blue compartment, it returns to the perforated wall (in the frames around the middle of Fig. 2a, part of the red compartment can be seen to the right of the fish). However, instead of passing through to the red compartment the fish instead turns back and remains in the blue compartment. Figure $2 \mathrm{~b}$ shows the trajectory of the zebrafish. The red and blue dots denote the zebrafish's locations in the red and blue compartments, respectively. Using this information, four indices ((a-d) listed below) could be calculated to describe zebrafish activity. (a) Mean velocity: the average swimming speed across both compartments (in trials involving uniform illumination) or in each individual compartment (in colour preference trials); (b) Velocity Distribution (VD): the distribution of a zebrafish's transient swimming speed in one or both compartments. Given that a fish may swim or rest, depending on the environmental condition, a simple average may not adequately represent its pattern of swimming behaviour and hence velocity distribution provides a more comprehensive description of changes in swimming speed; (c) Compartment Occupied Ratio (COR): the time ratio a zebrafish stays in one compartment relative to the other. This is a particularly important index. A zebrafish is likely to stay in the compartment illuminated with the colour it prefers, or leave the compartment illuminated with the colour it dislikes. An alteration in the zebrafish's vision may change its colour preference and hence its behaviour; (d) Through Holes Count per Thousand Seconds (TH): the average number of times a zebrafish passes through the holes in the perforated wall during each trial period. The higher the value of $\mathrm{TH}$, the more times the fish moves between compartments. If the zebrafish is active, it is likely to travel between the two compartments more frequently. 


\subsection{Histology and immunohistochemistry}

For Haematoxylin and Eosin staining the fixed eyes were dehydrated in a series of alcohol concentrations, embedded in paraffin and sectioned in a microtome at $7 \mu \mathrm{m}$ thickness.

222 Sections were rehydrated and stained in Haematoxylin and Eosin solutions and pictured using an Olympus camera. For immunostaining, fixed eyes were cryo-protected in $20 \%$ sucrose, embedded in Cryomatrix medium (VWR, UK) and quickly frozen using dry ice. $10 \mu \mathrm{m}$ sections were cut in a cryomount at $-20^{\circ} \mathrm{C}$, then mounted on superfrost slides and air dried at room temperature for at least 30 minutes prior to storage at $-80^{\circ} \mathrm{C}$. Slides were thawed at room temperature for 20 minutes and washed three times in phosphate buffered saline (PBS). Then the sections were blocked using blocking buffer (5\% sheep serum and $2 \%$ bovine serum albumin in PBS containing $0.3 \%$ Triton X-100) for 1 hour at room temperature. Sections were washed once with PBS and incubated in primary antibodies anti-4D2 (1:400) or antiZPR-1 (1:500) (monoclonal from ZIRC) in PBS with $0.1 \%$ Triton X-100 at $4{ }^{0} \mathrm{C}$ overnight. Sections were then washed twice with PBS and incubated with secondary antibody (Alexa fluor 488 anti-mouse (1:500), Molecular Probes) for 2 hours at room temperature. Finally, sections were washed three times with PBS and nuclei stained and mounted with DAPI (1.5 $\mu \mathrm{g} / \mathrm{ml}$ ) mounting medium (Vectashield Limited) and imaged using Ziess LSM 510 confocal microscopy.

\subsection{Data analysis}

Data from each experiment were compared using an ANOVA. The level of significance was set at $\mathrm{p}<0.005$. All data were presented as mean \pm standard error of the mean (SEM).

\section{Results}


244 Eighteen 8-month-old zebrafish were tested under uniform white illumination. Six fish were tested in each of three brightness conditions (10, 150 and 350 lux). They were placed individually in each compartment (right or left alternately) at the beginning of each trial.

247 Since the ambient illumination of the two compartments was the same, all indices were calculated for the zebrafish behaviour across the entire tank rather than for the individual compartments. In the low brightness environment (10 lux) mean velocity was $25 \mathrm{~mm} / \mathrm{s}$; in both of the brighter environments (150 and 350 lux) mean velocity increased to $50 \mathrm{~mm} / \mathrm{s}$ (Fig. 3A-C). Compared to both the 10 lux and 150 lux conditions, TH was significantly higher under 350 lux illumination (Fig. 3D). However, given that VD under 150 lux had two peaks, 5 and $50 \mathrm{~mm} / \mathrm{s}$ (Fig. 3A-C), a distribution indicative of the burst-and-coast mode typical of adult zebrafish (Videler \& Weihs, 1982; Muller et al., 2000), an illumination level of 150 lux was used for subsequent parts of this study.

\subsection{The influence of colour of uniform ambient illumination on the swimming behaviour of} untreated and acrylamide-treated zebrafish

The swimming behaviour of 8-month-old untreated $(n=18)$ and acrylamide-treated zebrafish $(n=12)$ was tested under uniform coloured ambient illumination. In each condition (white, red and blue) the ambient colour of the two compartments was identical, as was the brightness (150 lux in all instances). As can be seen from the data in Fig. 4A-C, VD changed quite markedly under different ambient colour illumination and between untreated and treated fish. With the white illumination, the velocity distribution of untreated zebrafish had two peaks ( 5 and $50 \mathrm{~mm} / \mathrm{s}$ ); under all other conditions (untreated fish in red and blue uniform lighting; treated fish in all conditions) the distribution had a single peak. The mean velocity in each of the colour environments was significantly reduced following acrylamide exposure 
(Fig. 4A'-C'). Apart from this reduction in swimming speed, all fish treated with acrylamide showed no other abnormalities (such as freezing or zig-zagging) in their motor behaviour.

\subsection{Colour preference of untreated and acrylamide-treated zebrafish in different colour filter} pair environments

The colour preference of untreated and acrylamide-treated zebrafish was tested using three paired combinations of colour-illuminated environments: blue-red, blue-white and red-white. In every case the brightness of the ambient colour was 150 lux. The COR results for untreated zebrafish (Fig. 5A-C) indicate a marked colour preference: the fish preferred blue to red, blue to white, and white to red. In other words, the order of preferred colour was blue, white and red. The COR results for $2.0 \mathrm{mM}$ acrylamide-treated zebrafish hese fish remained in the compartment in which they were first placed and did not move to a compartment for which control fish had demonstrated a preference (Fig. 5A-C).

\subsection{Acrylamide treatment causes retinal damage in zebrafish}

To investigate the toxic effects of acrylamide on zebrafish retina, both histological and histoimmunostaining assays were carried out. Haematoxylin and eosin staining showed rod outer segments had almost disappeared in adult fish exposed for $36 \mathrm{hrs}$ to $2 \mathrm{mM}$ acrylamide. The number of rod nuclei was markedly reduced. The cones in the untreated retina were well aligned, while cones in the treated retina were disorganized. The total thickness of the photoreceptor layer was significantly decreased (Fig. 6A). Immunostaining with anti-4D2 antibody (labelling rod outer segments) revealed significantly shortened outer segments of rod cells (Fig. 6B). Immunostaining for arrestin 3a using anti-ZPR1 (labelling red and green double cones) showed the double cones were still present but the length of these cones was markedly reduced (Fig. 6C). These histological and histochemical analyses indicated that 
retinal structure was affected in the acrylamide-treated fish, but does not reveal the relation between the extent of retinal damage and the associated visual loss. Our colour-preferencebased behavioural technique allows the possibility of correlating structural changes with functional changes.

\section{Discussion}

The zebrafish has become an increasingly important model for the investigation of the genetic bases of degenerative diseases and the effects of environmental chemical factors. However, in order that genetic or environmental effects can be clearly elucidated it is necessary to establish a comprehensive description of the organism's normal behavioural repertoire. With regard to behaviours mediated by visual experience, determining typical zebrafish preferences for different levels of illumination, different colours and different patterned environments is an essential prerequisite for investigating functional changes in the zebrafish visual system. The methodology presented here utilised a colour preference method to characterise the vision of living zebrafish. Self-developed software allowing the analysis of locomotion was used to generate quantifiable indices of swimming behaviour influenced by the colour of the environment. Using this method, we detected changes in behavioural phenotypes under different test conditions and in fish treated with acrylamide, a substance with known neurotoxic effects. The experimental set-up allowed the zebrafish access to two linked compartments, each of which was illuminated independently by white, red or blue light; the zebrafish chose to spend the majority of its time in the "blue" compartment when the neighbouring compartment was "red" or "white", and the majority of its time in the "white" compartment when the neighbouring chamber was "red". More concisely, the order of zebrafish colour preference was blue, white and red. This is in keeping with the findings of Li et al. (2014) who used a place preference tank identical to that used in the present study 
(although they produced differently coloured compartments by placing coloured paper on the

319 floor of the separate chambers): they reported that the control zebrafish spent most time in blue and green compartments and least time in the red compartment. Similarly, BarbaEscobedo and Gould (2012) found that zebrafish were more likely to enter a blue rather than orange box (as assessed by initial preference) while Bault et al. (2015) found that zebrafish preferred colours of shorter wavelength. These and the current results differ from the findings reported by Avdesh et al. (2012) who used both a place preference procedure similar to that used in the current study and a T-maze procedure. The results from both procedures indicated that, of all colours used, red was most preferred while blue was least preferred. It is possible that the apparent disparity with the present results is due to the different methods used: in the place preference procedure used by Avdesh et al. (2012) the floor of the adjacent compartments was covered with coloured gravel that was illuminated by an unspecified light source, the characteristics of which may have influenced the zebrafish. Furthermore, with both procedures the various test areas were distinguished not only by differences in colour but differences in reflectance.

Our method not only allows objective recording and analysis of normal behavioural phenotypes but also assessment of behavioural changes resulting from environmental factors. Acrylamide is a neurotoxic agent known to cause retinal dysfunction and axonopathy in rats, cows and primates (El-Sayyad et al., 2011; Godin et al., 2000; Lynch et al., 1989; Wild \& Kulikowski, 1984; Merigan et al., 1982). In the current study, exposure to acrylamide produced measurable changes in zebrafish behaviour: following acute treatment with $2.0 \mathrm{mM}$ acrylamide, zebrafish no longer exhibited the colour preferences that had been shown by the untreated fish. While it is tempting to interpret these behavioural changes as a consequence of the structural changes in the zebrafish retina produced by acrylamide exposure (i.e. the cone cell disorganisation revealed by histological analysis), it is of course possible that the 
neurotoxic effects might have caused direct damage to the zebrafish motor circuitry. Li et al.

344 (2016) observed a significant reduction in aspects of locomotor behaviour in the nematode

Caenorhabditis elegans following exposure to acrylamide. However, the fact that in the current study the zebrafish exposed to acrylamide were still capable of locomotion, albeit at velocities that were reduced relative to those of the control group (Fig. 5), does suggest that the observed behavioural changes were unlikely to be due to motor deficits alone (although further work is required to ascertain if any sensory deficits are colour specific or merely due to changes in acuity).

Although the current paper describes the effects of only one particular chemical on zebrafish behaviour, the technique we have developed and the aspects of visual-preference behaviour we have defined offer a simple and straightforward method of assessing the effects of any toxicant, including pharmaceutical contaminants. In addition, while the majority of previous studies have focussed on pre-natal and early post-natal exposure (Pamanji et al., 2015; Sun et al., 2014), our technique allows evaluation of neurotoxic effects on the visionmediated behaviour of adult zebrafish. Furthermore, it offers a platform for investigating a wide spectrum of neurodegenerative disorders including, among many others, Huntington's Disease, Alzheimer's Disease and amyotrophic lateral sclerosis, all of which have been modelled in zebrafish (Kabashi et al., 2011), but also appraising possible genetic or chemical therapies that could be used in the treatment of these conditions and so providing a platform for preclinical drug development. More specifically, the technique's utility in evaluating functionality of the zebrafish visual system allows correlation of visual-related behavioural changes with the structural and functional changes revealed by other investigative approaches including electrophysiological and immunohistochemical methods. 
Research Trust, the Rosetrees Trust, and the Glasgow Children's Hospital Charity for supporting this work. The maintenance of GCU zebrafish facility was funded by the EU

INTERREG NEW noPILLS programme.

\section{References}

Avdesh, A., Martin-Iverson, M.T., Mondal, A., Chen, M., Askraba, S., Morgan, N., Martins,

R.N. (2012). Evaluation of color preference in zebrafish for learning and memory. Journal of Alzheimer's Disease, 28 (2), 459-469.

Barba-Escobedo, P.A. \& Gould, G.G. (2012). Visual social preferences of lone zebrafish in a novel environment: strain and anxiolytic effects. Genes, Brain and Behavior, 11, 366-373.

Bault, Z. A., Peterson, S. M., \& Freeman, J. L. (2015). Directional and color preference in adult zebrafish : Implications in behavioral and learning assays in neurotoxicology studies, Journal of Applied Toxicology, 35 (12), 1502-1510. potato chips on the development of retina in albino rats. Asian Pacific Journal of Tropical Biomedicine, 1 (4), 253-260.

Emran, F., Rihel, J., \& Dowling, J. E. (2008). A behavioral assay to measure responsiveness of zebrafish to changes in light intensities. Journal of Visualized Experiments, (20). pii: 923. genetics. Progres in Retinal and Eye Research, 27 (1), 89-110. 
392

393

394

395

396

397

398

399

400

401

402

403

404

405

406

407

408

409

410

411

412

413

414

415

Fleisch, V.C., \& Neuhauss, S.C.F. (2006). Visual behaviour in zebrafish. Zebrafish, 3 (2), 191-201.

Godin, A.C., Dubielzig, R.R., Giuliano, E., \& Ekesten, B. (2000). Retinal and optic nerve degeneration in cattle after accidental acrylamide intoxication. Veterinary Ophthalmology, 3 (4), 235-239.

Kabashi, E., Brustein, E., Champagne, N., \& Drapeau, P. (2011). Zebrafish models for the functional genomics of neurogenetic disorders. Biochimica et Biophysica Acta, 1812 (3), 335-345.

Li, H., Chuang, A.Z., \& O’Brien, J. (2009). Photoreceptor coupling is controlled by connexin 35 phosphorylation in zebrafish retina. The Journal of Neuroscience, 29(48), 15178-15186.

Li. J., Li, D., Yang, Y., Xu, T., Li, P. \& He, De. (2016). Acrylamide induces locomotor defects and degeneration of dopamine neurons in Caenorhabditis elegans. Journal of Applied Toxicology, $36(1), 60-67$.

Li, X., Liu, B., Li, X.-L., Li, Y.-X., Sun, M.-Z., Chen, D.-Y., Zhao, X. \& Feng, X.-Z. (2014). $\mathrm{SiO} 2$ nanoparticles change colour preference and cause Parkinson's-like behaviour in zebrafish. Scientific Reports, 4, 3810.

Lynch, J.J., Eskin, T.A., \& Merigan, W.H. (1989). Selective degeneration of the parvocellular projecting retinal ganglion cells in a New World monkey, Saimiri sciureus. Brain Research, $499(2), 325-332$.

Merigan, W.H., Barkdoll, E., \& Maurissen, J.P. (1982). Acrylamide-induced visual impairment in primates. Toxicology and Applied Pharmacology, 62 (2), 342-345.

Muller, U.K., Stamhuis, E.J. \& Videler, J.J. (2000) Hydrodynamics of unsteady fish swimming and the effects of body size: comparing the flow fields of fish larvae and adults. Journal of Experimental Biology, 203 (2), 193-206. 
416 Newman, M., Verdile, G., Martins, R. N., \& Lardelli, M. (2011). Zebrafish as a tool in

417 Alzheimer's disease research. Biochimica et Biophysica Acta, 1812 (3), 346-52.

418 Pamanji, R., Bethu, M.S., Yashwanth, B., Leelavathi, S. \& Venkateswara Rao, J. (2015).

419 Developmental toxic effects of monocrotophus, an organophosphorous pesticide, on

420 zebrafish (Danio rerio) embryos. Environmental Science and Pollution Research, 22 (10),

$421 \quad 7744-7753$.

422 Parng, C., Roy, N. M., Ton, C., Lin, Y., \& McGrath, P. (2007). Neurotoxicity assessment using zebrafish, Journal of Pharmacological and Toxicological Methods, 55 (1), 103-112.

Pérez-Escudero, A., Vicente-Page, J., Hinz, R. C., Arganda, S. \& de Polavieja, G. G. (2014). idTracker: tracking individuals in a group by automatic identification of unmarked animals. Nature Methods, 11 (7), 743-748.

Raghupathy RK, McCulloch DL, Akhtar S, Al-Mubrad TM, Shu X. (2013) Zebrafish model for the genetic basis of X-linked retinitis pigmentosa. Zebrafish, 10(1):62-69

Schloss, K. B. (2015). Color Preferences Differ with Variations in Color Perception. Trends in Cognitive Sciences, 19 (10), 554-555. enantiospecific differences of two $\beta$-blockers, propranolol and metoprolol, in the embryos and larvae of zebrafish (Danio rerio). Environmental Toxicology, 29 (12), 1367-1378. Videler, J.J., \& Weihs, D. (1982). Energetic advantages of burst-and-coast swimming of fish at high speeds. Journal of Experimental Biology, 97 (1), 169-178.

Wild, H.M., \& Kulikowski, J.J. (1984). Neurotoxic effects of acrylamide on rat retinogeniculate fibres. Behavioural Brain Research, 13(3), 201-207. 
442 Figure 1 Schematic representation of the tank and the setup used for behavioural analysis.

443 The tank was divided into two connected compartments of equal size, separated by an 444 opaque, perforated wall. Illumination came from an LED panel placed underneath the tank. 445 Coloured filters could be placed between the underside of the tank and LED panel. A shade was placed around the LED light panel to block any extraneous light. The LED panel light was powered by an adjustable DC supply. A digital video camera connected to a laptop was placed above the tank to record the swimming behaviour of the fish.

Figure 2 Locomotion and trajectory of adult zebrafish in red-blue tank compartments. (a) A sequence of clips from the captured video. Time flows from left to right, top to bottom. Clips in second row from column ten to fifteen shows the movement of zebrafish from the red to the blue compartments. (b) Representative trajectory of zebrafish movement / swimming pattern of zebrafish in red compartment and blue compartment; the darker sections of the red and blue lines correspond to the period shown in (a).

Figure 3 Velocity distributions (VD) of 8-month-old zebrafish under illumination of 10 lux under different levels of illumination (D). Error bars represent the standard error of the mean. TH was significantly higher (**) under 350 lux compared both to 10 and 150 lux $(\mathrm{p}<0.001)$.

Figure 4 Velocity distributions (VD) of 8-month-old untreated and 2mM acrylamide (ACR) treated zebrafish under uniform ambient colour illumination. (A) VD in uniform white illumination; (B) VD in uniform blue illumination; (C) VD in uniform red illumination, showing the distribution of untreated and $2 \mathrm{mM}$ ACR treated fish. In all cases the brightness 
was 150 lux. A', B' and C' shows the mean velocities of untreated and $2 \mathrm{mM}$ ACR treated

467 fish. Error bars represent the standard error of the mean. Velocity of the $2 \mathrm{mM}$ ACR treated fish in white, red and blue light illumination was significantly reduced compared to the untreated fish $(\mathrm{p}<0.0001)$.

Figure 5 Compartment occupancy ratio (COR) for 8-month-old untreated and $2 \mathrm{mM}$ acrylamide (ACR) treated zebrafish in different ambient 150 lux colour pair combinations: (A) red-blue (R-B); (B) white-red (W-R); (C) white-blue (W-B). *represents the starting compartment of the zebrafish. Error bars represent the standard error of the mean. Significant differences $(\mathrm{p}<0.0001)$ are represented by $* * * *$.

Figure 6 Haematoxylin and Eosin (H\&E) staining and immunostaining of retinal sections of untreated and $2 \mathrm{mM}$ acrylamide (ACR) treated zebrafish. (A) H\&E stained retinal sections shows the affected rod outer segments (ROS) of ACR-treated zebrafish. (A') Thickness of the photoreceptor layer relative to the whole retina (expressed as \%). ACR treated zebrafish exhibited significantly shorter photoreceptor layer $(* * *, p=0.0005)$. Error bars represent the standard error of the mean. (B) Immunostaining the retinal sections with anti-4D2 antibody shows the localisation of rhodopsin in the ROS. Prominent and uniform rhodopsin localisation was observed in the ROS of the untreated zebrafish retinal sections, but abnormal localisation and pattern was observed in the retinal sections of ACR treated zebrafish. Nuclei of ONL, INL and GCL were labelled by DAPI staining. (B') Thickness of rod outer segments (ROS) relative to the whole retina (expressed as \%). ACR treated zebrafish had shorter ROS when compared untreated controls $(* *, p=0.0014)$. Error bars represent the standard error of the mean. (C) Anti-ZPR1 staining shows the localisation of arrestin 3a in the red and green cone photoreceptors. ZPR1 immunostaining shows shorter / abnormal double cones in 

the ACR treated zebrafish. Nuclei of ONL, INL and GCL were labelled by DAPI staining.

492

493

494

495

496

497

498

499

500

501

502

503

504

505

506

(C') Thickness of double-cone layer relative to the whole retina (expressed as \%). Double cones were significantly shorter than that of untreated controls $(* * * *, p<0.0 .0001)$. Error bars represent the standard error of the mean. RPE, retinal pigment epithelium cells; ROS, rod outer segments; ONL, outer nuclear layer; INL, inner nuclear layer; GCL, ganglion cell layer.

Figure 1
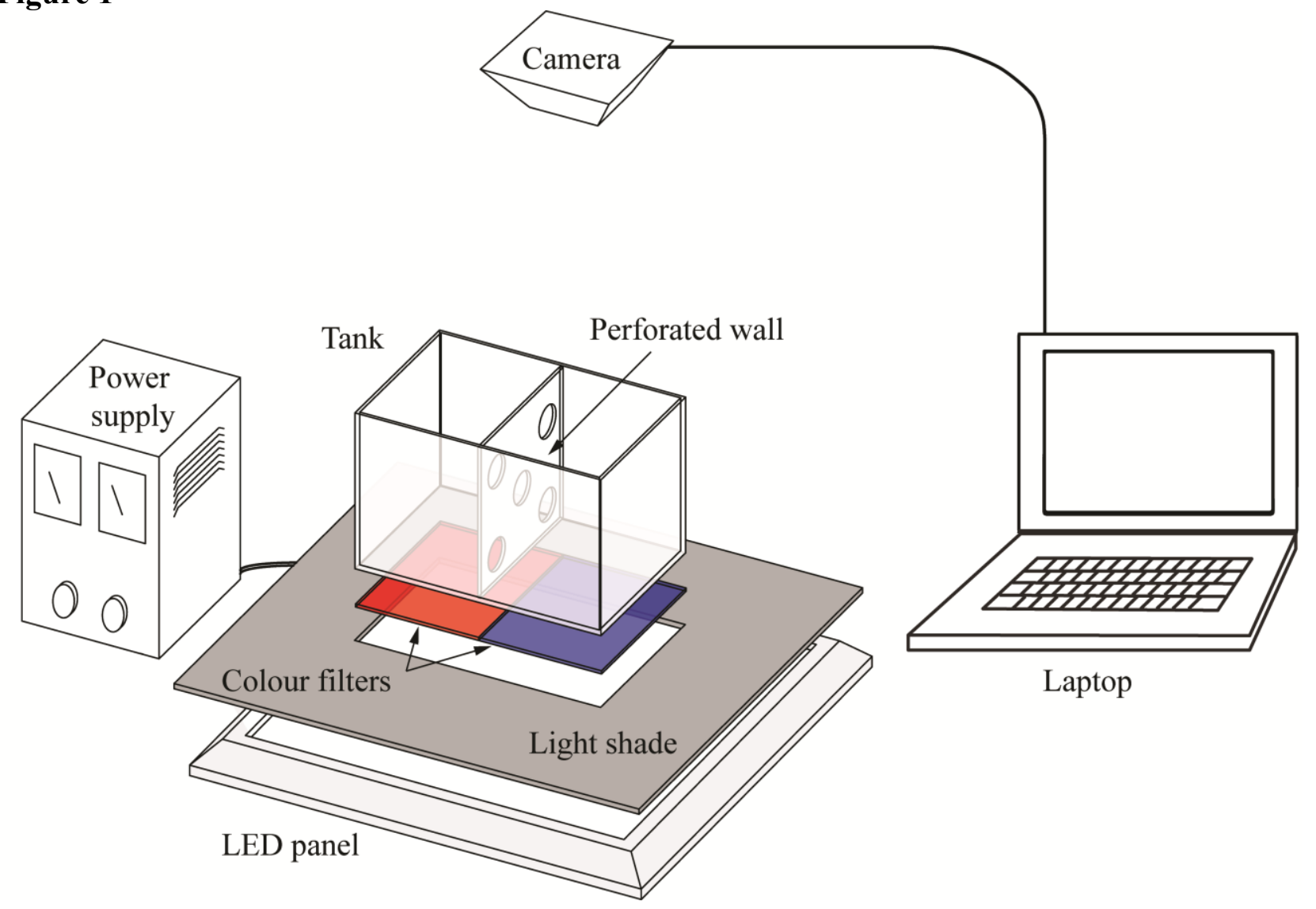

Figure 2 
(a)

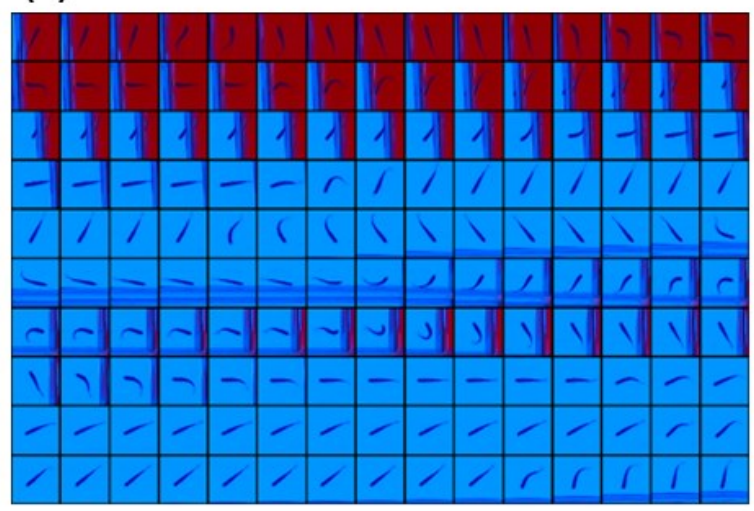

511

512

513

514

515

516

517

518

519

520

521

522

Figure 3 (b)

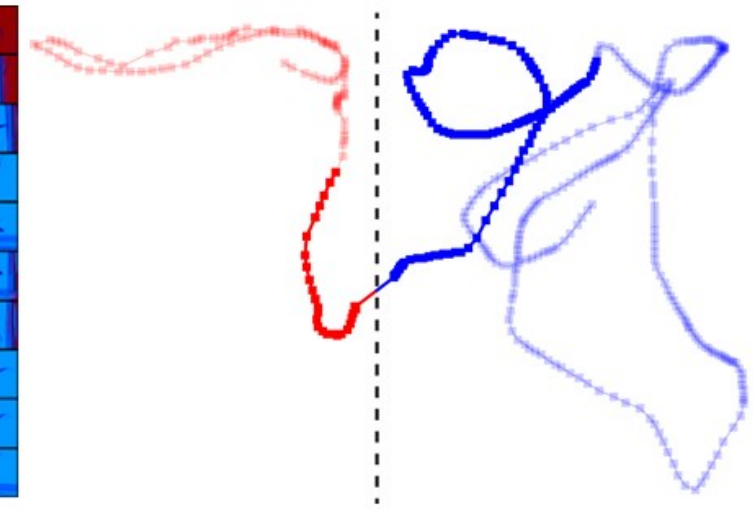

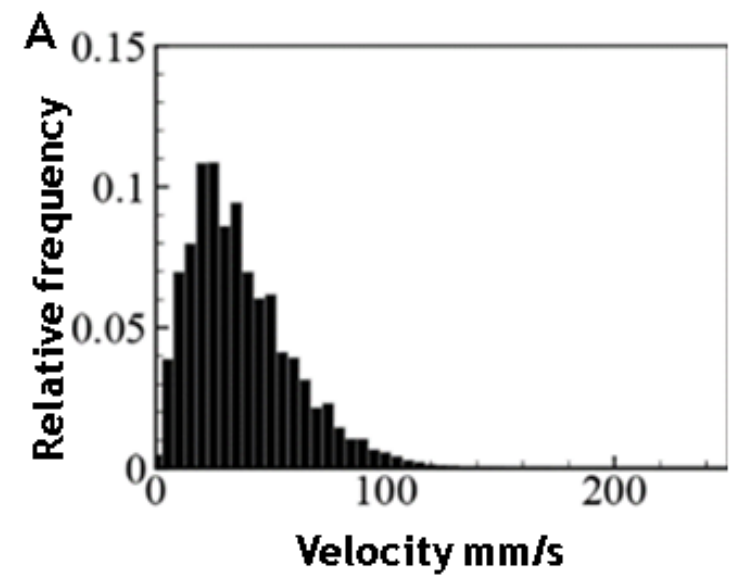
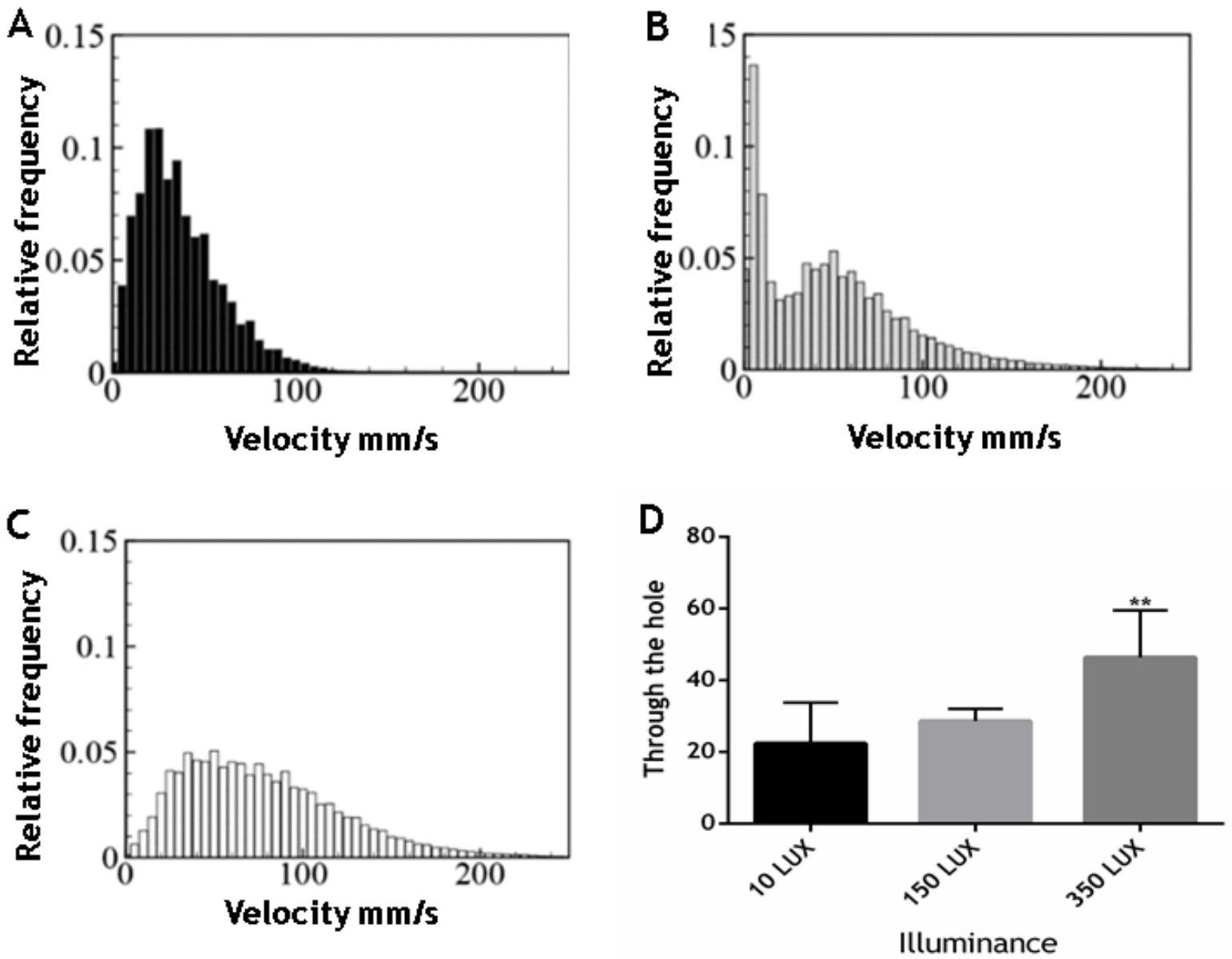
Figure 4
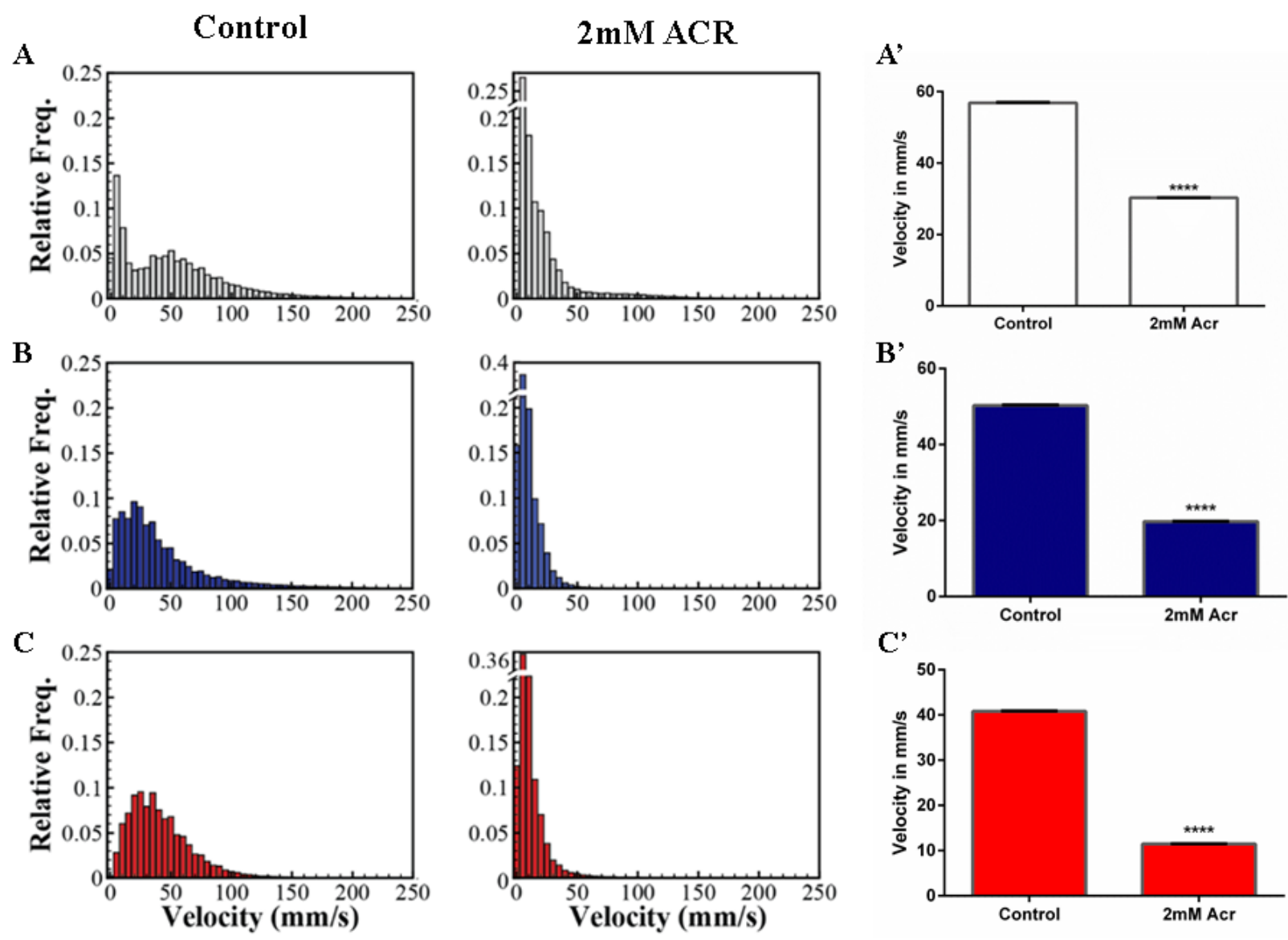
573 Figure 5A

A Untreated

(a)

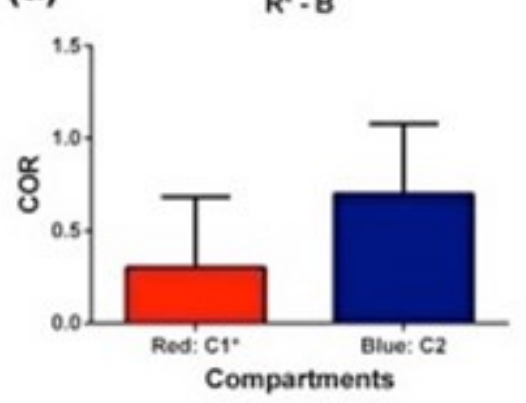

(c)

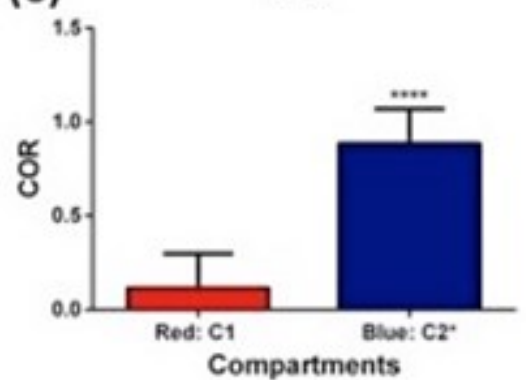

574

\section{Figure 5B}

\section{2mM Acr}
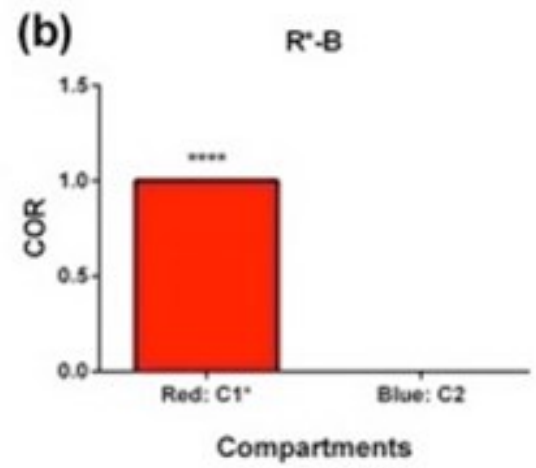

(d) R-B

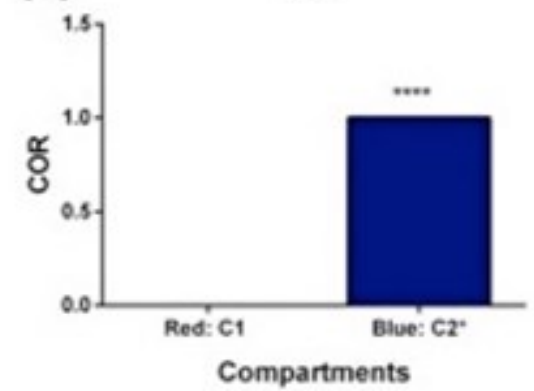

$\square$ Red: C1

Blue: C2
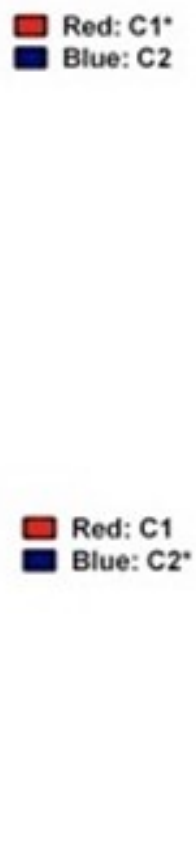
B Untreated

(a)

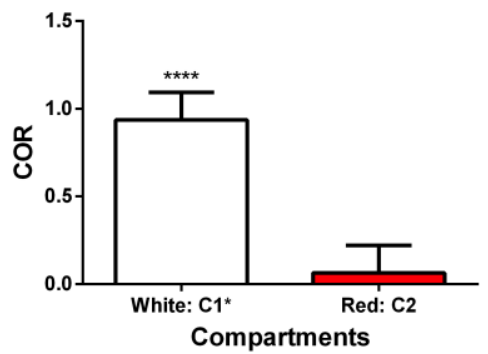

(c)

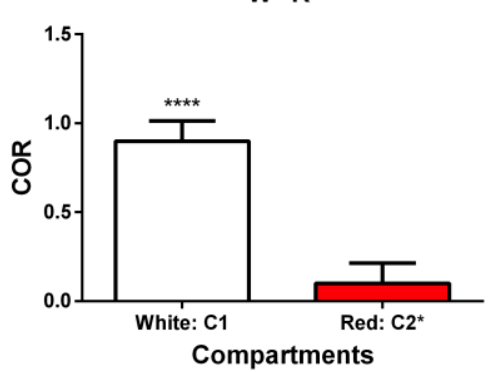

2mM Acr

(b) $\quad w^{*}-R$

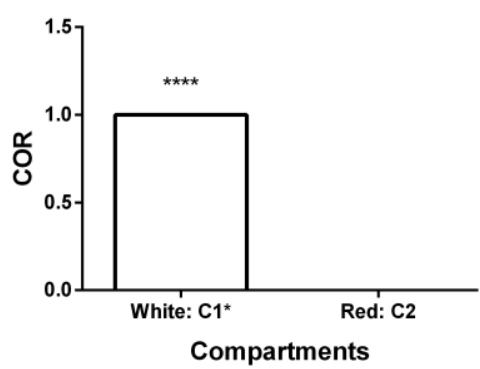

(d) $\quad \mathrm{W}-\mathrm{R}^{\star}$

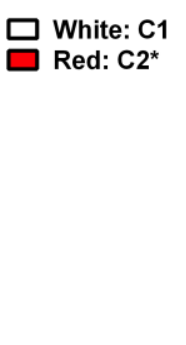

$\square$ White: C1

Red: C2

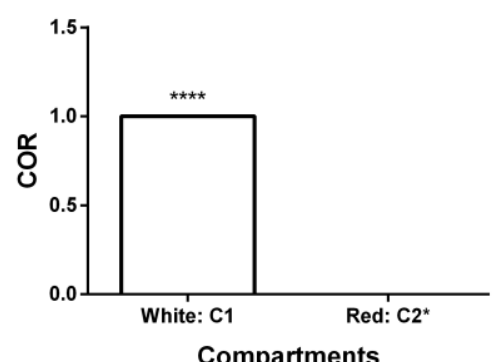

$\square$ White: C1

Red: C2

$\square$ White: C1

Red: C2*

\section{Figure 5C}


C Untreated

(a)

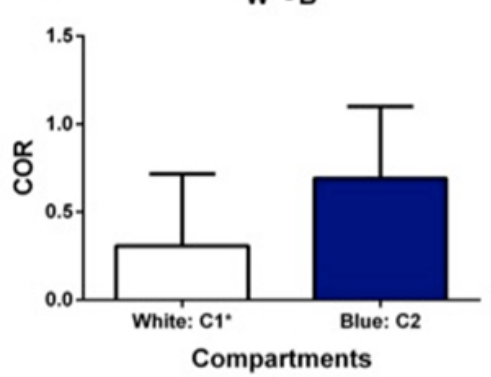

(c)

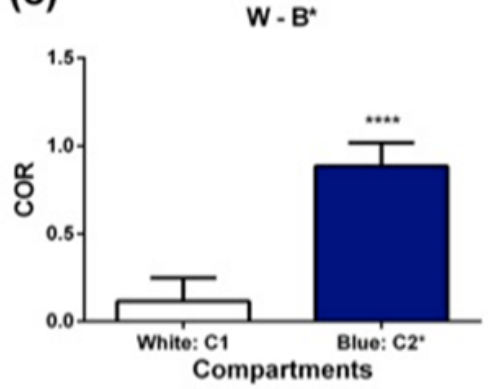

2mM Acr

(b)

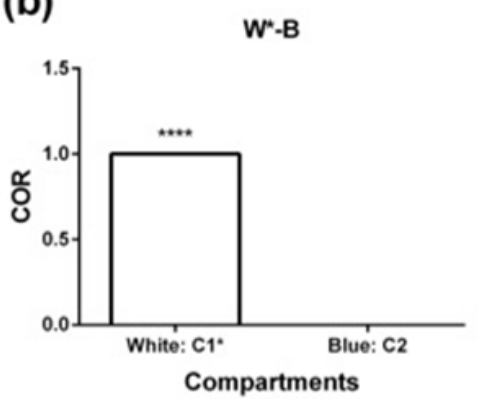

(d) W-B*
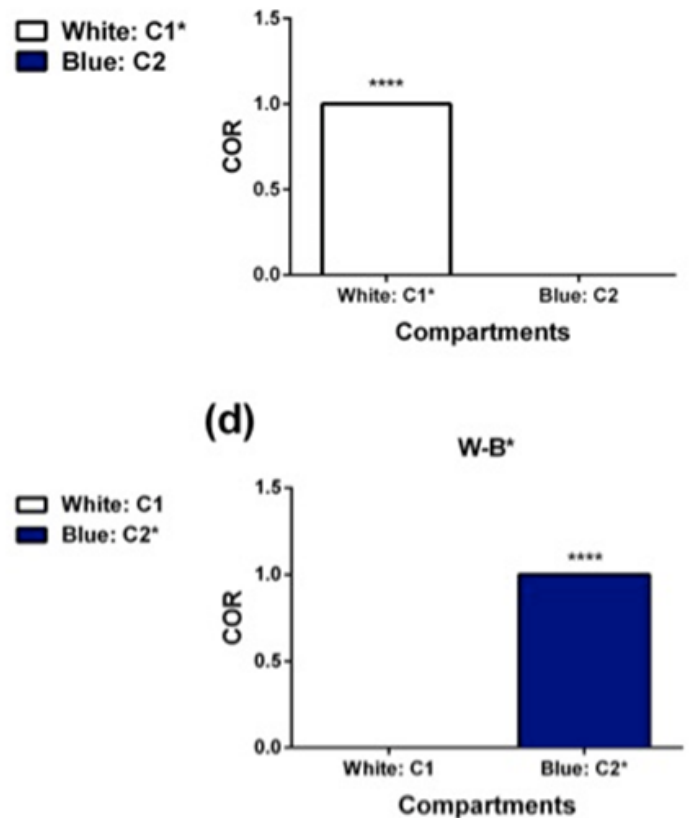

$\square$ White: C1

Blue: C2 
A

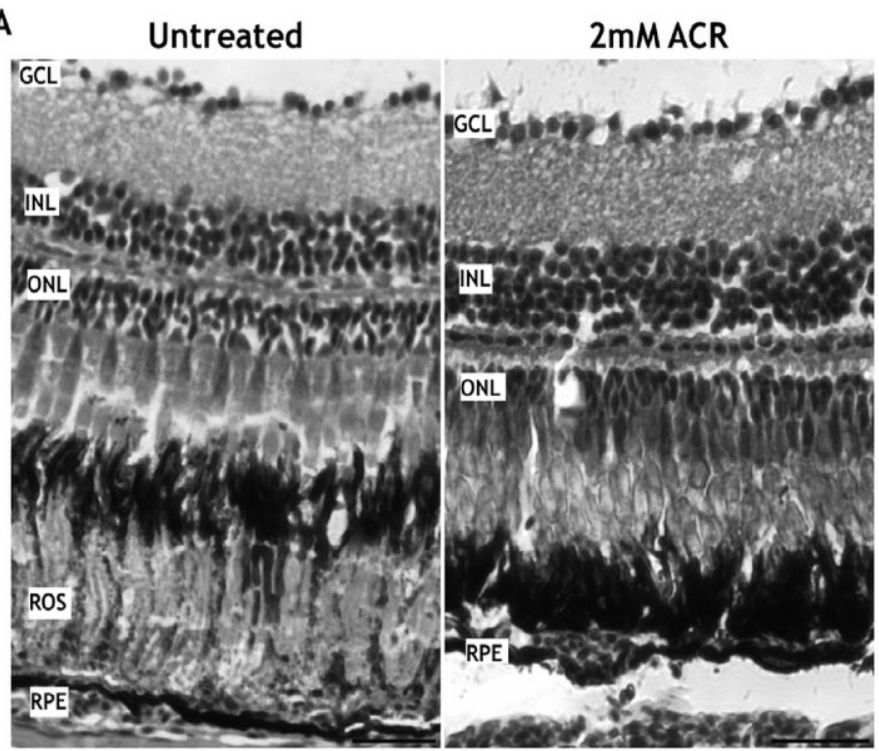

B

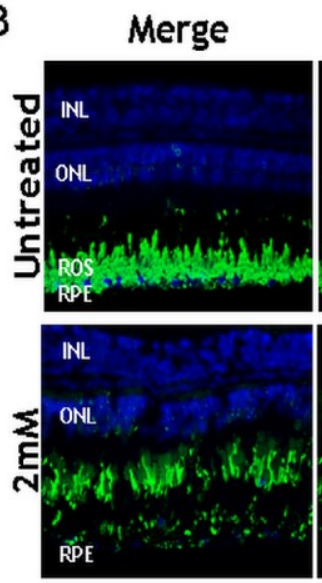

C Merge

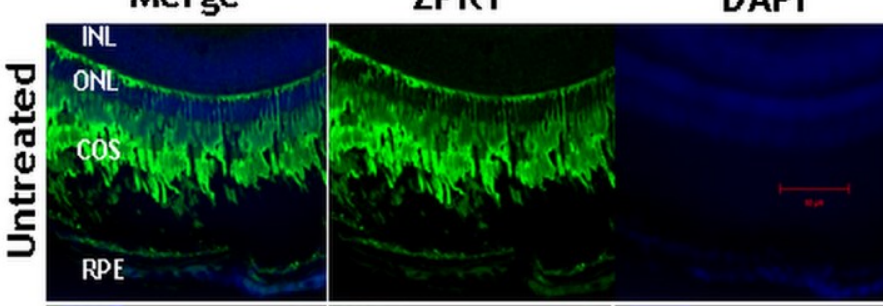

हี

610

611
4D2
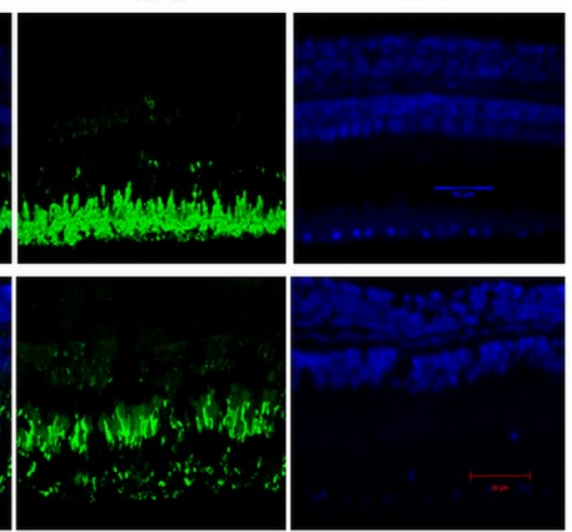

DAPI

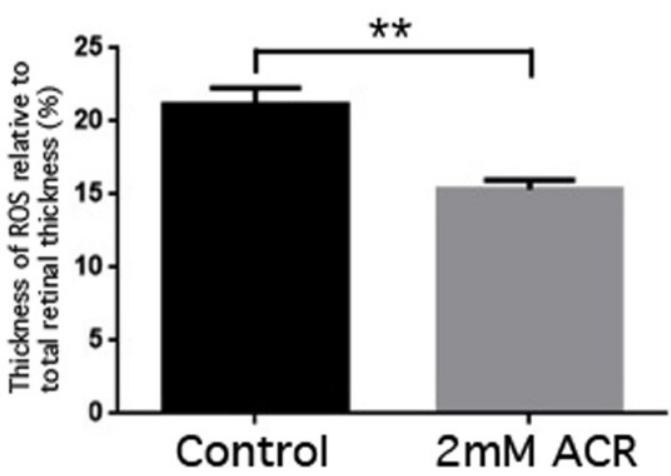

$c^{\prime}$

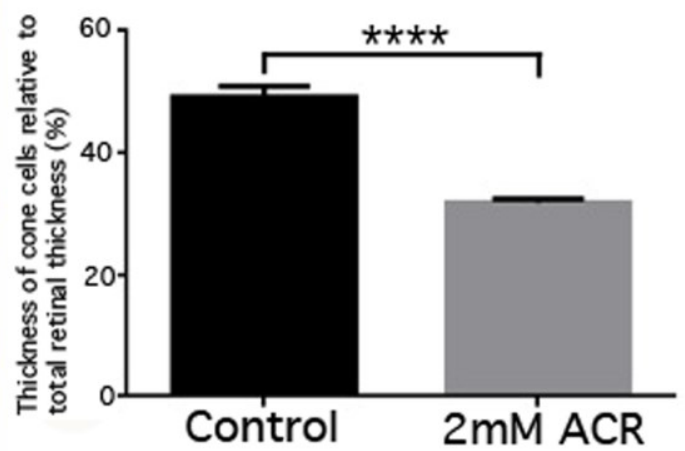

